\title{
Student Astronomical Research in Hawai'i - A Case Study For Use of Robotic Telescopes In Education
}

\author{
J. D. Armstrong ${ }^{1 *}$, Mary Kadooka ${ }^{2}$, Carolyn Kaichi ${ }^{3}$, Geoff Mathews $^{4}$, Michael Nassir $^{2}$, Donn \\ Starkey ${ }^{5}$
}

\begin{abstract}
The arrival of robotic telescopes has increased productivity among astronomers and opened fields to people who previously would not have been able to participate. Since the advent of robotic telescopes, a new generation of astronomers has started to arise. A generation of astronomers in their teens, freed from late night observing and data processing, are now making scientific contributions to the field alongside professional astronomers. We present some of the award winning work being done by young astronomers in high school and middle school in Hawai'i using the innovation of robotic telescopes.

${ }^{1}$ University of Hawaii Institute for Astronomy, Maui, Hawaii

${ }^{2}$ University of Hawaii Department of Physics, Oahu, Hawaii

${ }^{3}$ University of Hawaii Institute for Astronomy, Hilo, Hawaii

${ }^{4}$ Foothill College, Los Altos Hills, California

${ }^{5}$ DeKalb Observatory, Auburn, Indiana

*Corresponding author: jd@ifa.hawaii.edu
\end{abstract}

\section{Background and Introduction}

There has been a growing recognition of the importance of genuine inquiry for learning the process of science, as presented in the National Science Education Standards for Content, "Science As Inquiry" (Singer 2005). That report identified a number of science learning goals that have been attributed to laboratory experiences, including "mastery of subject matter; developing scientific reasoning; understanding the complexity and ambiguity of empirical work; developing practical skill; understanding the nature of sciences; cultivating interest in science and interest in learning science; and developing teamwork abilities." Genuine research projects help students to achieve the learning objectives compared to traditional classroom laboratory experiments that may not engage students in the scientific decision-making process.
Current recommended practices in science teaching, from the National Research Councils' A Framework for K-12 Science Education (Schweingruber et al., 2012), are modeled on the practices of working scientists. They have identified 8 core practices: "1. Asking questions... 2. Developing and using models, 3. Planning and carrying out investigations, 4. Analyzing and interpreting data, 5. Using mathematics and computational thinking, 6. Constructing explanations... 7., Engaging in argument from evidence, 8. Obtaining, evaluating, and communicating information."

The scientific inquiry process is too complex, however, for students to carry out without instruction. A key element of effective instruction is scaffolding (Bruner 1974, Puntambekar and Hubscher 2005), in which students iteratively practice a skill with progressively less teacher support. This practice helps keep students operating in the zone of proximal development 
(Vygotsky, 1980), a state where they are challenged enough to maintain learning without being overwhelmed by tasks that are too difficult.

Several works have described similar scaffolding methods to introduce students to the practices of scientific inquiry (e.g., Bell et al. 2005, Dunkhase, 2003). Bell et al. (2005) describes a simplified model of the steps of scientific inquiry: question generation, methodology, and solution, as well as a fading process that successfully scaffolds student mastery of these practices (see Bell et al. 2005, Fig. 2). Initially, the instructor models a full inquiry by providing questions, methodology, and the approach to finding a solution to students. In a second iteration of the inquiry process, the instructor provides a question and methodology for students, but lets students determine their own method for generating a solution. In a third iteration, students also determine their methodology, and in a fourth, students also generate their question.

These iterations are referred to as Confirmatory, Structured, Guided, and Open Inquiry. Not all instruction progresses students through all four stages of inquiry.

Fitzgerald et al. (2014) provided an inventory of projects that provide students with opportunities to engage with the astronomy research process at a range of levels, and classifies the dominant mode used by each project. While most of the programs described in Fitzgerald et al. (2014) have students carry out a single project under either a Structured or Guided inquiry model, some do iterate through progressive stages of inquiry instruction. Instruction begins with structured inquiries that serve to teach core astronomy knowledge and analysis skills, proceeds to guided inquiries, and culminates with matching students with mentors as they carry out open inquiries.

In this contribution, we discuss the role that robotic telescopes have played in enabling Open Inquiry by high school students working with astronomers at the University of Hawaii at Manoa (UH), primarily through involvement with the Hawaii
Student Teacher Astronomical Research program (HI STAR).

\section{HI STAR}

The HI STAR program (Garland et al., 2008) is a one-week summer camp held annually at the University of Hawai'i at Mānoa (with one year, 2015, at UH Maui College). Students from O'ahu, Maui, Kaua'i, Moloka'i, and Hawai'i Island who were entering the 8th through 12th grade have attended the program, many for multiple years. The program of the camp is designed to teach the students research skills, particularly in the area of astronomy. Following the camp, students are paired with mentors with the goal that the students will work on a meaningful research project for entry in the science and engineering fair.

Over the last 10 years UH has worked with over 120 pre-college aged students with the goal of helping those students develop scientific literacy. The vast majority of these students have connected with the university through HI STAR, though a few have independently matched with a mentor.

The stated goals of HI STAR are:

1. Students will develop the skills and knowledge base for doing scientific inquiry and conducting rigorous, real-world investigations in astronomy.

2. Students will complete appropriate, rigorous and real-world empirical research in astronomy for an authentic audience.

3. Students will improve upon oral and written communication skills in order to share their science and will develop a supporting and collaborative network of peers, mentors, and the general public.

The one-week camp is designed to provide students with background knowledge in astronomy as well as to replicate the experiences which a professional astronomer might encounter in his/her career. UH faculty and staff give lectures on the process skills (photometry, astrometry, etc.) used in the research, 
fundamental physics and astronomy (nature of light, gravity, etc.) as well as research topics the students could pursue (asteroids, exoplanets, variable stars, galaxies, etc.) These experiences tend to follow the Structured model of inquiry, where students are explicitly taught processes and skills yet are still coached through final analyses.

During the course of the one-week camp the students are placed in groups with similar research interests. These groups work with a mentor to produce a small body of research, typically following a Confirmatory or Structured model of inquiry. The research project is presented at the end of the program to the other students in the program. The research project is also presented to professional researchers and the general public in a setting similar to that of a professional research conference.

After the camp, students are paired with long term mentors to carry out a project somewhere on the spectrum of Structured, Guided, or Open inquiry. The students are given the goal of carrying out research for entry in the local science and engineering fair. The local Science and Engineering Fairs are held annually in the spring, which gives students several months to perform a serious study. The Fairs have a structure that approximates a poster session at a professional astronomical conference.

Example one-week project: Discovery of potential variable young stars. A team of students were provided with a set of observations of 4 young stars known to have circumstellar disks of potentially planet-forming material. The observations consisted of blue (B), green (V), red (R), and near-infrared (I) filter observations of a wide field around the target stars. Multiple observations were carried out in each filter over a week in the month preceding the HI STAR camp.

The students compared the brightness of their target stars to nearby stars that were known to not be variable, and looked for evidence that their brightness varied. From this search, they identified one that varied in brightness by more than $5 \%$ during the observation period, and another that varied by $\sim 1 \%$, marking these as candidates for more detailed follow-up. The students were able to obtain further observations from the Las Cumbres Observatory network (LCO, discussed in more detail below) during their one-week project, allowing for rapid confirmation of their initial findings. Further work may lead to determining the nature of the variability - star spots, accretion events, or other mechanisms.

Example one-week project: Determining the age of globular clusters. A team of students were provided with a set of observations of a globular cluster. The observations consisted of multiple images taken in B, V, R, and I filters. The students were also provided with access to a resource to generate isochrones ${ }^{1}$.

The students stacked multiple observations in each filter. The magnitudes of several hundred stars were measured for each of the filters. Color magnitude diagrams were generated, and compared to theoretical isochrones. For this project standard star observations were not available. The magnitudes were left in instrumental units. It was shown that even without calibrated magnitudes, it was possible to accurately determine ages.

Example long term project: Follow-up of exoplanet candidates and observations of candidate exoplanets identified by the CoRoT satellite (Bordé et al., 2003). The CoRoT mission identified transient events with high precision photometry. However the instrument had an angular resolution of 15 " X 25 ". Higher resolution observations were needed to identify the source of the transient event and confirm the observation.

Observations of predicted transit events were obtained using the LCO. Students performed the photometry and constructed light curves for the observations. The students also performed some analysis of the transient events i.e. determining if a transit is observed, transit depth and other features such as asymmetry. The observations (images) were shared with professional astronomers who

\footnotetext{
${ }^{1}$ The BaSTI web tools http://albione.oa-teramo.inaf.it/main_mod.php
} 
then performed independent analysis. The Students interacted with their mentor. The mentor interacted with the science team. Effectively the professional and student researchers operated in parallel with the mentor as the common element. The project resulted in the confirmation of an exoplanet.

Example long term project: Search for large rapidly rotating asteroids. Asteroids with a diameter of greater than 100 meters generally rotate with a period of greater than 2.4 hours. The reason is that asteroids larger than 100 meters in diameter are generally rubble piles. They are composed of several pieces held together by gravity. Assuming a density similar to water it can be shown that gravity alone is insufficient to hold together an asteroid which rotates faster than once every 2.4 hours.

The student used the LCO to obtain observations of several large asteroids with unknown rotation rates. Photometry was performed on the observations and the light curve was constructed. It was then shown that the asteroids in the sample all rotated lower than the 2.4 hour cutoff period. The student also included the calculations which showed that gravity alone is insufficient to hold together asteroid which rotates faster than the 2.4 hour cutoff period. The project is valuable in classifying asteroids. It is important to know if an asteroid which could potentially hit the earth is a rubble pile or a monolith.

\section{HI STAR Metrics}

The primary metric which we use for evaluating the HI STAR program is the number of students entering local science and engineering fairs. Approximately $60 \%$ of the HI STAR alumni students entered local science fairs. During the first 10 years of the HI STAR program (the years for which the science fairs have been held as of the time of writing this paper), nearly 100 projects were completed. 16 of the projects were completed in areas outside of astronomy (including engineering), or were 'report type' projects. 2 projects were of a theoretical nature. Of the remaining projects, about half used robotic telescopes; specifically the LCO network and half used archival type data (including data acquired by the student's mentor.)

While not part of our primary metric, we have also tracked some of the honors they have received.

Approximately two dozen students have advanced all the way to the Intel International Science and Engineering Fair, and at least three projects have been awarded third place in category. The students have won or been offered over $\$ 750,000$ in scholarships and awards at the science and engineering fairs. We do not stress awards for the students, but instead encourage students to value the scientific research process.

In addition to the science fair, students are also encouraged to participate in science beyond the science and engineering fair. In particular, they are encouraged to attend the Pacific Symposium of Science and Sustainability. Students from the program have been co-authors on two peer-reviewed papers (Cabrera et al. 2015 and Caballero et al. 2013). Furthermore, an external review of the HI STAR program conducted in 2014 (Davis, 2014) found that students gain interest and efficacy in the area of scientific inquiry.

\section{Application of Robotic Telescopes}

From our experiences with HI STAR and as astronomy researchers, we identify four primary modes of obtaining data for projects:

1. Classical: In the classical mode of data acquisition, the researcher travels to the observing site and operates the equipment in person. This mode may include projects in which the researcher constructs special equipment for his/her observations.

2. Remote Observing: In the remote observing mode the telescope is operated from a remote site in a real time mode.

3. Robotic: We define a robotic observation as one where the observations are preprogrammed, and it is not necessary for 
the researcher or telescope operator to have real time interactions with the observatory.

4. Archival: Archival data is data which is taken without the request of the researcher. It may include observations which were taken for another project, or it may be data which was taken for a regular observing campaign. Surveys such as the SDSS, the AAVSO variable star database and PanSTARRS fall in this category, as do observations from solar observing campaigns such as the SOHO. (All of these archives have been used by student researchers in our programs.)

Classical, Remote, and Robotic observing modes all allow for the observer to set parameters such as target, filter, integration time, and cadence, while Archival observations call for the observer to find pre-existing data that matches their needs.

As part of their lease, the Las Cumbres Observatory (LCO) has an operating agreement with the University of Hawai'i in which they provide time on the LCO network to UH. This time is intended for education and outreach programs such as HI STAR. (For a description of the LCO network see Brown et al. 2013, and Shporer et al. 2010). The LCO can be thought of a single observatory with a distributed site. Telescopes on the network do not operate independently. In the current mode of operation, observations are submitted for a specific class of telescope and instrument, as well as observing window. The network then assigns observations to individual telescopes. It is only possible to stipulate a specific telescope or observing site with extra effort on the part of the observer.

In the early years of the HI STAR program the LCO consisted of only the 2.0 meter Faulkes Telescope North on Haleakala and the (nearly) identical Faulkes Telescope South at Siding Springs, Australia. The telescopes could be operated as robotic telescopes or in a "Real Time Interface" (RTI) mode, which in our terminology is remote observing. The latter option is no longer available on the network.
During the first year of the program, students only obtained data using the RTI. In the second and subsequent years, an astronomer was added to the team who learned how to use the telescope in the robotic mode. Once the option was added it was found that obtaining observations through a robotic mode was generally preferred due to time savings for the individual student researchers. However, there is an overhead cost to the project due to time needed to program the telescope observations. While it was possible to teach each of the student researchers to use the telescopes in robotic mode, the time required to train the individual students and the difficulty of managing multiple accounts was impractical.

Introductory observing experiences with the RTI were carried out at the one-week camp until the RTI interface at Faulkes telescopes was discontinued in 2012. The experience of controlling the telescope live was seen as a way to increase the student's connection to the data. This made the science more "real" and provided the students with a higher degree of ownership in their project. Students who attended the HI STAR camp after 2012 were not able to experience advantages of the Faulkes RTI.

However, since 2008, HI STAR students have been able to use a $0.41 \mathrm{~m}$ amateur telescope via RTI. The telescope, DeKalb Observatory (MPC Code: H63), is located in Indiana. The students are able to remote control the telescope under the tutelage of the telescope operator, who is located at the telescope. One advantage to the students is the +6 hour time differential between Hawai' $i$ and Indiana - e.g., 10pm local in Indiana is 4pm local in Hawai'i.

We note that there were no projects from students in the HI STAR program in which the students took data in a classical manner on the telescopes. While there is a selection bias; the bias has mainly been due to practical concerns: Access to observing time was not likely available to the students, except on the LCO network. More importantly, for students to observe on moderate to large size telescopes would require the students to travel to the 
telescopes.

There was one case (Armstrong and Tong, 2016) in which the student approximated observations in a classical mode. The student was an intern working on Maui as part of the Maui Economic

Development Board's STEMworks ${ }^{\mathrm{TM}}$ program. The student (Tong) went to the summit of Haleakala and operated the Faulkes Telescope North from across the road using the Real Time Interface. While this data was not used in the resulting paper, the student reported that observing at the summit was the highlight of the internship experience.

\section{Discussion}

\section{Advantages and Disadvantages of Robotic Telescopes in Education}

From our work with HI STAR students, we have identified numerous advantages and disadvantages of robotic telescopes. Advantages include:

1. Robotic telescopes are more efficient at acquiring observations, requiring less time to obtain the same observations with the same telescope. This is due to the delay between the completion of the last observation and the operator beginning subsequent observations.

2. Robotic telescopes and remote observatories eliminate travel time and costs of going to the observatory. In our case many of the students live on different islands and would need to fly to the island that hosts the observatory.

3. Observations with robotic telescopes can be programmed at times that are more reasonable for students. Astronomical observations are generally taken at night. (Solar observations, radio astronomy, and remote observations are notable exceptions.) Keeping student researchers up all night requires the following day for students to recover. Thus, daytime work is generally better received by parents of the students.
4. Robotic telescopes and remote observatories provide access to parts of the sky which might not be accessible, and also to observing times which might not be accessible. In Hawai' $i$, access to the LCO has provided students with a convenient way to observe targets which would otherwise be too far south to observe locally. For observations with time constraints - such as observing exoplanet transits - student researchers have a larger range of observing windows to observe, including the opportunity to get coordinated observations from multiple sites. This option can allow for extended observations; possibly even 24 hour (or longer) coverage of an event.

5. Robotic telescopes and archival data provide an ease of monitoring. Observations can be scheduled to occur for a few minutes periodically over a potentially long time span.

Some of the disadvantages of robotic telescopes include:

1. The students do not develop an understanding of the challenges associated with obtaining data. An example is that when weather interferes with observations, the students may become frustrated, not understanding that astronomy is generally subject to weather.

2. Students do not develop as strong a connection to the observations. This connection can result in a greater understanding of the subject of study. We find the disconnect is greatest for archival data, less intense for robotic observing, even less for remote observing. We find that the greatest connection to the subject is gained when the student researcher is able to see with their own eyes - by looking at the object with the unaided eye, or by looking through an eyepiece. This does not generally result in measurable observations yet has a valuable engagement effect. 
3. The use of robotic telescopes requires greater care and experience for planning the observations. Once observations begin with a robotic telescope changes are generally not made. Unless someone is monitoring the observations, over/under exposure and other miscalculations are likely to persist through the entire observing run. It is strongly advised that an experienced observer work with student researchers using robotic systems.

\section{Robotic Telescopes Effectively Scaffold Methodology}

As an illustration of the use of robotic telescopes in scaffolding, we consider a student project determining the age and distance to an open star cluster. Distances are determined by measuring apparent brightness of the stars and comparing to the modeled brightness of stars at a given distance (usually 10 parsecs). In order to obtain the most accurate measurement of the brightness of stars, it is necessary to compare the observations of the target stars to well calibrated reference stars. The observations should be made under the same sky conditions and at the same latitude in the sky. The former is accomplished by observing the standards then the targets and then re-observing the standard stars. This process requires observational planning beyond the ability of beginning students in our program.

The steps of scaffolding could be for the student to first determine times during which the target is available. After the student is able to determine target visibility windows, the student was asked to determine altitudes of the target at the time of observation. The student can then learn how to select reference stars, and determine the best times to observe the reference stars and targets.

Programming these observations can be tricky and is left as the final step.

The case of HI STAR illustrates how robotic telescopes support scaffolding the learning of astronomical research skills. The mathematics involved in planning telescope observations include e.g., spherical geometry, with relatively complicated calculations involving the latitude and longitude of observatories, algebra involving functions with many variables for calculating observing times based on target magnitudes, telescope size, and detector wavelength-dependent sensitivities, along with read-noise and other uncertainty sources. The reduction of imaging data from telescopes requires computers to load large arrays and carry out several mathematical operations to e.g., flat-field and dark subtract, along with the need of applying somewhat advanced mathematics to achieve accurate coordinate solutions. Learning this set of skills is typically reserved to a graduate-level astronomical techniques course or on-the-job training for graduate students.

The availability of finished data - photometric measurements and object positions - allows students to focus their efforts on the key analysis steps - the sense-making.

In the Bell et al. (2005) nomenclature, robotic telescopes allow students to bypass the methodology of measurement, to focus on the more interesting methodology of analysis (which includes the transferable skills developed by looking for patterns and carrying out statistical analysis). This can also allow them to more quickly reach the stage of developing their own questions, which is the most scientifically rich aspect of the scientific process.

\section{Acknowledgements}

The authors would like to thank the Air Force Office of Scientific Research, Maui Economic Development Board, DeKalb Observatory, and the Las Cumbres Observatory for their support of this work.

\section{References}

Armstrong, J. and Tong, W. (2016). Accuracy and Precision of Multicolor Observations of Four Double Stars. Journal of Double Star Observations, 12:28-31. 
Bell, R. L., Smetana, L., and Binns, I. (2005). Simplifying inquiry instruction. The Science Teacher, 72(7):30-33.

Bordé, P., Rouan, D., and Léger, A. (2003). Exoplanet detection capability of the COROT space mission. Astronomy \& Astrophysics, 405(3):1137-1144.

Brown, T., Baliber, N., Bianco, F., Bowman, M., Burleson, B., Conway, P., Crellin, M., Depagne, É., De Vera, J., Dilday, B., et al. (2013). Las Cumbres Observatory global telescope network. Publications of the Astronomical Society of the Pacific, 125(931):1031.

Bruner, J. S. (1974). From communication to language-A psychological perspective. Cognition, 3(3):255-287.

Caballero, R., Genet, R., Armstrong, J., McGaughey, S., Krach, C., Leatualli, A., Rohzinski, A., Rohzinski, C., Rohzinski, E., Rohzinski, N., et al. (2013). Six Proper Motion Pairs Measured with the 2-meter Faulkes Telescope North. Journal of Double Star Observations, 9(1):61-68.

Cabrera, J., Csizmadia, S., Montagnier, G., Fridlund, M., Ammler-von Eiff, M., Chaintreuil, S., Damiani, C., Deleuil, M., Ferraz-Mello, S., Ferrigno, A., et al. (2015). Transiting exoplanets from the CoRoT space mission-XXVII. CoRoT28b, a planet orbiting an evolved star, and CoRoT29b, a planet showing an asymmetric transit. Astronomy \& Astrophysics, 579:A36.

Davis, H. (2014). 2014 HI STAR Evaluation. TLC, Inc. Unpublished.

Dunkhase, J. A. (2003). The coupled-inquiry cycle: A teacher concerns-based model for effective student inquiry. Science Educator, 12(1):10.

Fitzgerald, M. T., Hollow, R., Rebull, L. M., Danaia, L., and McKinnon, D. H. (2014). A review of high school level astronomy student research projects over the last two decades. Publications of the Astronomical Society of Australia, 31.
Garland, C., Kadooka, M., Starkey, D., and Nassir, M. (2008). HI STAR: Building Bridges Between AAVSO Observers and High School Students. Journal of the American Association of Variable Star Observers (JAAVSO), 36:220.

Puntambekar, S. and Hubscher, R. (2005). Tools for scaffolding students in a complex learning environment: What have we gained and what have we missed? Educational psychologist, 40(1):1-12.

Schweingruber, H., Keller, T., Quinn, H., et al. (2012). A framework for K-12 science education: Practices, crosscutting concepts, and core ideas. Tech. Rep.

Shporer, A., Brown, T., Lister, T., Street, R., Tsapras, Y., Bianco, F., Fulton, B., and Howell, A. (2010). The LCOGT network. Proceedings of the International Astronomical Union, 6(S276):553555.

Vygotsky, L. S. (1980). Mind in society: The development of higher psychological processes. Harvard university press. 